\title{
MICROSTRUCTURE, MACROSTRUCTURE, AND MODELLING OF THE CENTRIFUGAL SPRAY DEPOSITION OF LARGE DIAMETER NI SUPERALLOY PREFORMS
}

\author{
M D Barratt ${ }^{1}, Z_{\text {Shi }}{ }^{2}$, R M Ward ${ }^{1}, \mathrm{P} \mathrm{S} \mathrm{Grant}^{2}, \mathrm{M} \mathrm{H} \mathrm{Jacobs}^{1}, \mathrm{~J} \mathrm{Mi}^{2}$ \\ ${ }^{1}$ IRC in Materials Processing, School of Engineering, University of Birmingham, Edgbaston, Birmingham B15 2TT, UK. \\ ${ }^{2}$ Department of Materials, University of Oxford, Parks Road, Oxford OX1 3PH, UK.
}

Keywords: IN718, Spray Forming, Microstructure, Modelling

\begin{abstract}
Centrifugal spray deposition (CSD) is being investigated for the production of ring-shaped preforms using Ni superalloys for aerospace applications. Experiments with different metal flow rate and different substrate movements have been performed to investigate the effects on the macro and microstructure of the resulting ring-shaped preforms. Modelling of heat flow during deposition was also performed to better understand the heat transfer and solidification behaviour of preforms. The mass flow rate and substrate reciprocation strongly influenced the micro- and macrostructure of the preforms. 'Hot' deposition conditions produced coarse microstructures, with interdendritic Laves phase and solidification shrinkage; 'cold' deposition conditions produced a banded equiaxed grained microstructure containing layering, prior droplet boundaries, and inter-splat porosity; and intermediate deposition conditions produced a good surface finish, equiaxed grain morphology, and reduced porosity. Heat flow modelling linked these microstructural observations to both short time scale re-heating/re-melting phenomena, and longer time scale behaviour related to the local solidification time and distribution of solid fractions. The as-sprayed preforms required a modification to standard heat treatment procedures, and produced a microstructure comprising fine-scale carbides, carbonitrides, and equiaxed grains.
\end{abstract}

Introduction

Centrifugal spray deposition was originally developed by Professor A.R.E Singer in the 1970s [1], whereby liquid metal is poured through a nozzle and directed onto a rapidly rotating disk or cup. At the edge of the disc, the liquid metal is atomised to form a spray that travels outwards and onto the inner surface of a reciprocating cylinder-shaped substrate where it solidifies to form a ring-shaped preform. The fundamental advantage of CSD over other spray forming routes is the mechanical production of the spray, without the need for expensive gas, allowing the process to be operated under vacuum or reduced pressure [2, 3]. Yield losses due to over spray are relatively low and overspray powder can be recycled as there is no gas pick-up [4]. Furthermore, porosity in the as sprayed preform will not contain gas and consequently will not reappear following thermomechanical processing. The process is ideally suited for the production of ring shaped components.

The spray forming facility at Birmingham University (Fig. 1) comprises a main melt chamber containing an induction heated bottom pour tundish furnace with a melt capacity of up to $50 \mathrm{~kg}$ of Ni alloy. The melting facility was designed to be operated in an inert gas or vacuum environment at pressures from $10^{-3}$ mbar to 1.1 bar. The centrifugal atomisation is achieved using a watercooled copper disc that rotates at speeds $>5000 \mathrm{rpm}$. The substrate motion is computer controlled, allowing the reciprocation pattern to be programmed precisely.

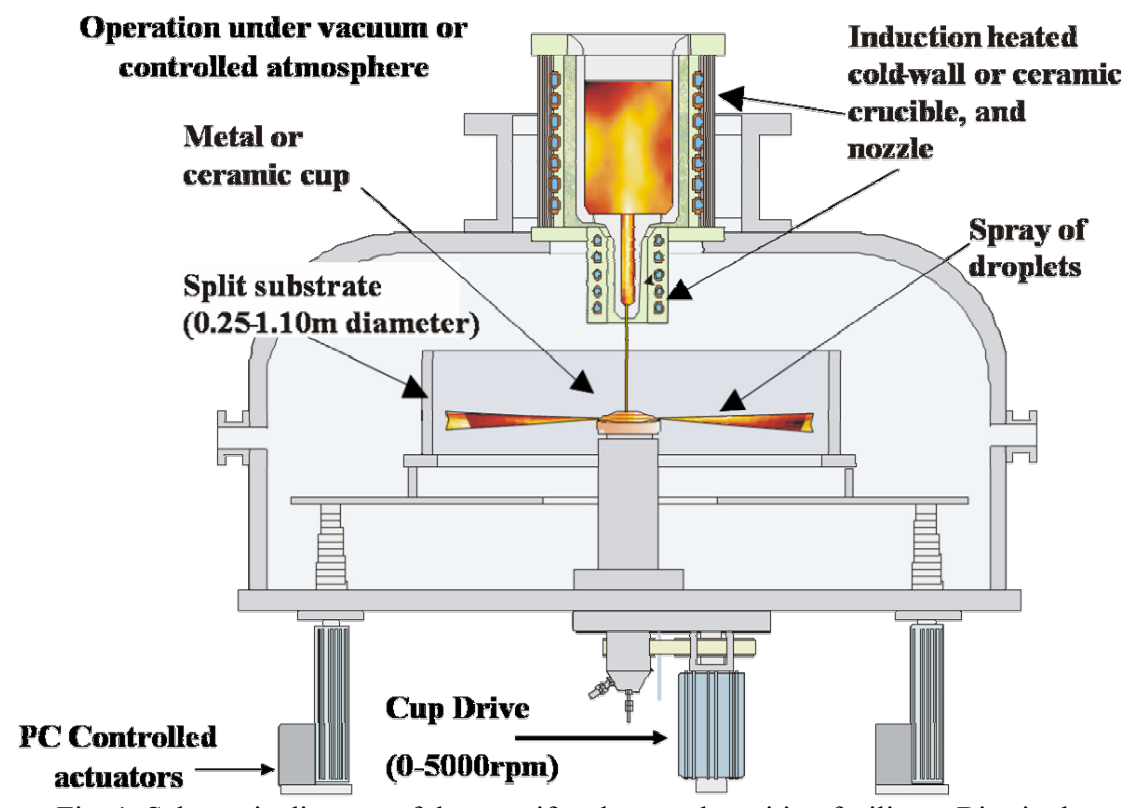

Fig. 1. Schematic diagram of the centrifugal spray deposition facility at Birmingham 
A UK Engineering and Physical Sciences Research Council (EPSRC) and Defence Science and Technology Laboratory (DSTL) funded research project is underway involving Birmingham University and Oxford University, together with Doncasters plc, Siemens, Bodycote Ltd, and Qinetiq. The objective of the project is to optimise the spray forming parameters via experiments and process modelling in order to manufacture reproducibly high quality $\mathrm{Ni}$ superalloy ring-shaped preforms with the geometry and macro/microstructure appropriate for thermo-mechanical processing into ring components.

In this study, ring-shaped preforms of IN718 have been produced using different processing conditions and the macrostructure and microstructure analysed. Heat treatment effects have also been investigated. Heat flow in the preform and substrate during deposition has been modelled using a finite difference (FD) method and results have been used to help explain the CSD microstructure. IN718 has a nominal composition of $18 \mathrm{Fe}, 18 \mathrm{Cr}$, $5 \mathrm{Nb}, 3 \mathrm{Mo}, 1 \mathrm{Ti}, 0.5 \mathrm{Al}, 0.04 \mathrm{C}, 0.004 \mathrm{~B}$ and balance $\mathrm{Ni}$ [5], and contains two main strengthening phases: gamma double prime $\left(\gamma^{\prime \prime}\right)$; and gamma prime $\left(\gamma^{\prime}\right)$ which produce coherency strains in the $\gamma$ matrix [5]. During high temperature aging treatments and sufficiently long times at temperatures above $650^{\circ} \mathrm{C}, \gamma^{\prime \prime}$ is replaced by delta phase. This has a similar composition to $\gamma^{\prime \prime}$ and grows as globular precipitates at the grain boundaries or as Widmanstatten plates. The globular grain boundary precipitates are important for controlling grain size during thermomechanical processing. However, the plate morphology is detrimental to the strength of the material by virtue of the aspect ratio of the precipitates [5].

\section{Experimental}

IN718 was processed by CSD using controlled mass flow rate and extent and rate of substrate motion. Nozzles with different diameters were used to vary the metal flow rate and a cylindershaped substrate with an inner diameter of $340 \mathrm{~mm}$ was employed to produce axi-symmetric ring shaped preforms. Other parameters such as cup geometry and rotation speed, melt superheat, and droplet throw distance were kept constant. Three experiments were performed using the following parameters:

(1) Hot deposition conditions: A 5.0mm nozzle diameter (mass flow rate of $0.257 \mathrm{~kg} . \mathrm{s}^{-1}$ ) and a reciprocation rate of $24.4 \mathrm{~mm} . \mathrm{s}^{-1}$ over an axial substrate displacement of $100 \mathrm{~mm}$.

(2) Cold deposition conditions: A $3.0 \mathrm{~mm}$ nozzle diameter (mass flow rate of $0.086 \mathrm{~kg} . \mathrm{s}^{-1}$ ) and an increased axial displacement of $125 \mathrm{~mm}$.

(3) Intermediate deposition conditions: A $3.0 \mathrm{~mm}$ nozzle diameter (mass flow rate of $0.092 \mathrm{~kg} . \mathrm{s}^{-1}$ ) a reciprocation rate of $7.81 \mathrm{~mm} . \mathrm{s}^{1}$, and a reciprocation displacement of $125 \mathrm{~mm}$.

In all cases, the cup rotation speed was maintained constant at $\sim 5000 \mathrm{rpm}$ and both melting and spraying were carried out under soft Ar vacuum of $\sim 3.0 \times 10^{-2}$ mbar.

Some as-sprayed ring samples were heat treated in accordance with two procedures:

(1) Standard procedure: solution treated and double aged $-1 \mathrm{hr}$ at $980^{\circ} \mathrm{C}$, air cool, $8 \mathrm{hrs}$ at $720^{\circ} \mathrm{C}$, furnace cooling at $50{ }^{\circ} \mathrm{C} \mathrm{hr}^{-1}$ to $620^{\circ} \mathrm{C}$, then $8 \mathrm{hrs}$ at $620^{\circ} \mathrm{C}$, air cool.

(2) Solution treated for $1 \mathrm{hr}$ at $1040^{\circ} \mathrm{C}$, followed by (1).

\section{Experimental Results}

Microstructural Evolution in CSD Processed IN718 Preforms

$\underline{\text { Hot Deposition Conditions }}$

Fig. 2(a) shows a slumped preform with poor shape and an unsatisfactory surface finish. The as-cast microstructure shown in Fig. 2(b) was coarse and consisted of dendrites and interdendritic regions containing $\mathrm{Nb}$ and $\mathrm{Ti}$ rich carbides/carbonitrides and a high volume fraction of Laves phase. $\mathrm{Nb}$ rich Laves phase is detrimental to the mechanical properties of IN718 because of both the plate-like nature of the precipitates and the $\mathrm{Nb}$ denuding effects on $\gamma^{\prime \prime}$ precipitation. Interdendritic regions also contained solidification shrinkage porosity, identified by the curved and irregular shaped pores.

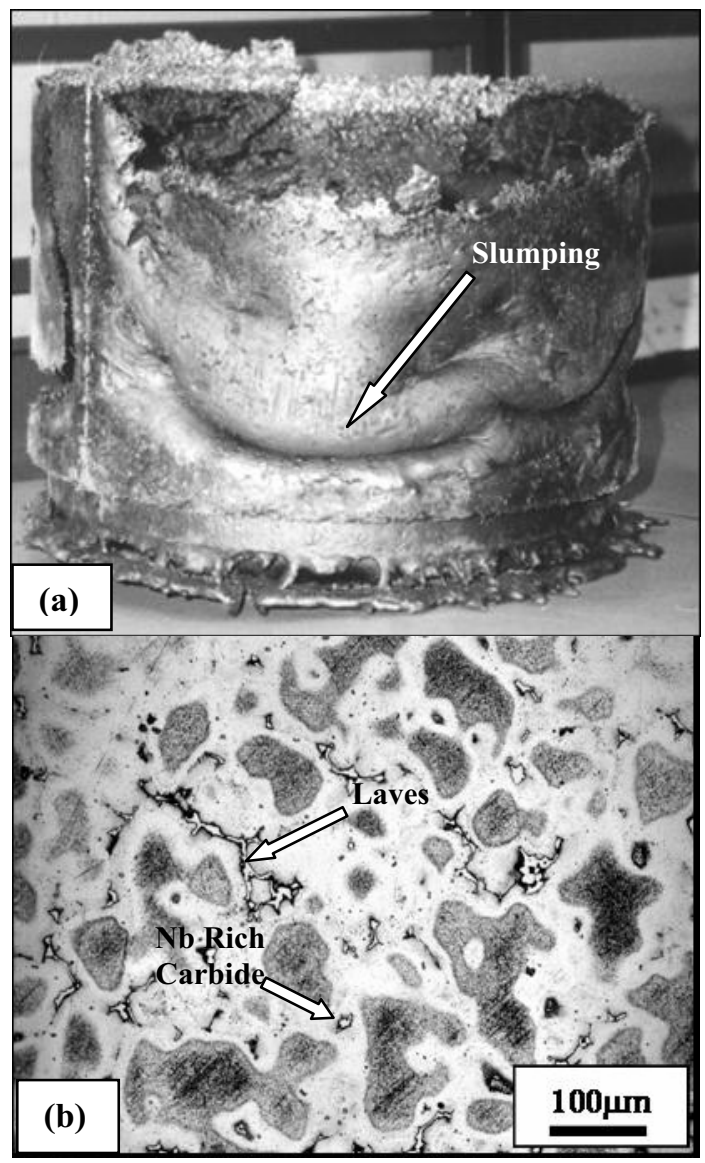

Fig. 2. (a) Photograph of a slumped ring produced under hot deposition conditions, and (b) a typical microstructure from a hot deposit.

Energy dispersive X-ray (EDX) microanalysis was carried out on the interdendritic region shown in Fig. 3(a). Elemental concentrations at each of the individual spot analyses are shown in Fig. 3(b). Over the range of locations, the $\mathrm{Nb}$ concentration ranged from 8 to $30 \mathrm{wt} \%$, these upper concentrations confirming Laves phase, together with the large globular appearance of the precipitates. Further support was lent by the relatively high Mo concentrations accompanying $\mathrm{Nb}$, suggesting $(\mathrm{Ni}, \mathrm{Fe}, \mathrm{Cr})_{2}(\mathrm{Nb}, \mathrm{Mo}, \mathrm{Ti})[5,6]$. 

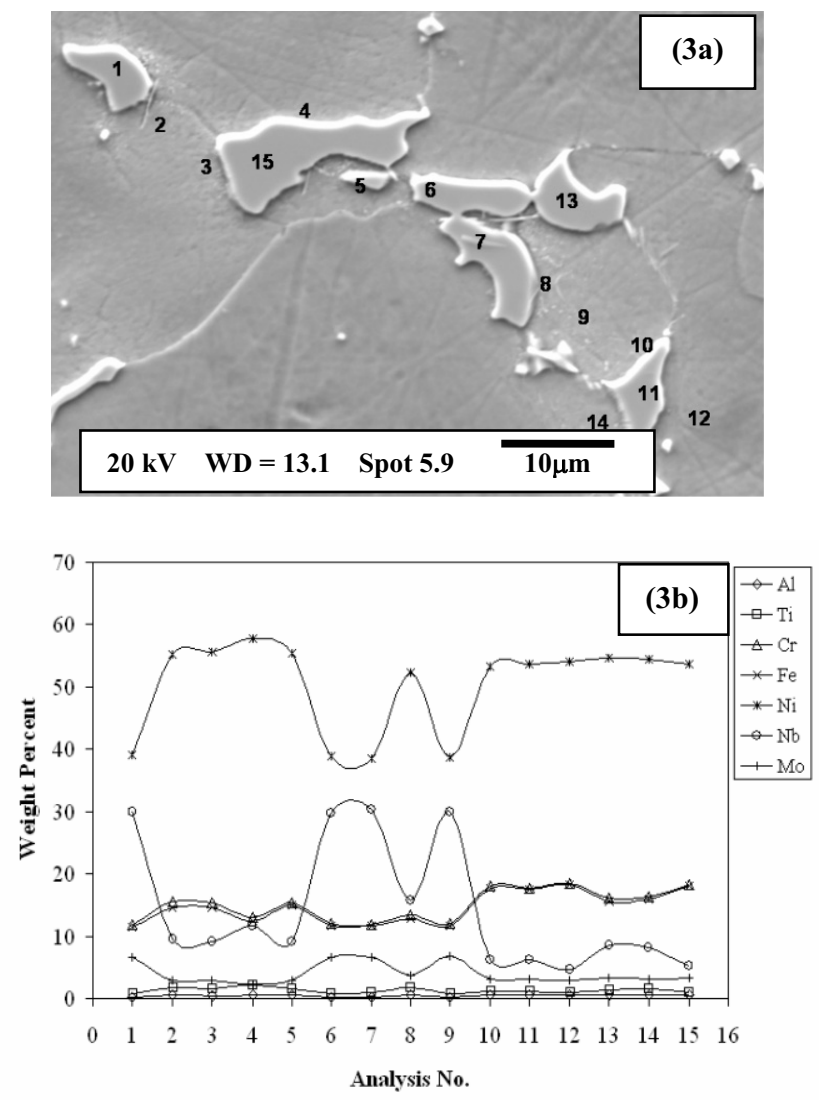

Fig. 3. (a) Secondary electron image of an interdendritic region in a hot deposit containing Laves phase, and (b) elemental concentration from EDX for the spot analyses in (a).

\section{$\underline{\text { Cold Deposition Conditions }}$}

Fig. 4(a) shows preform manufacture under cold deposition conditions, exhibiting poor shape, surface porosity and a low quality surface finish. The microstructure in Fig. 4(b) was banded, comprising inter-splat porosity, pancake-shaped grains and presolidified droplets. Banding, or layering, was more prominent at the outer and inner surfaces of the preform and aligned perpendicular to the direction of the incoming droplets.

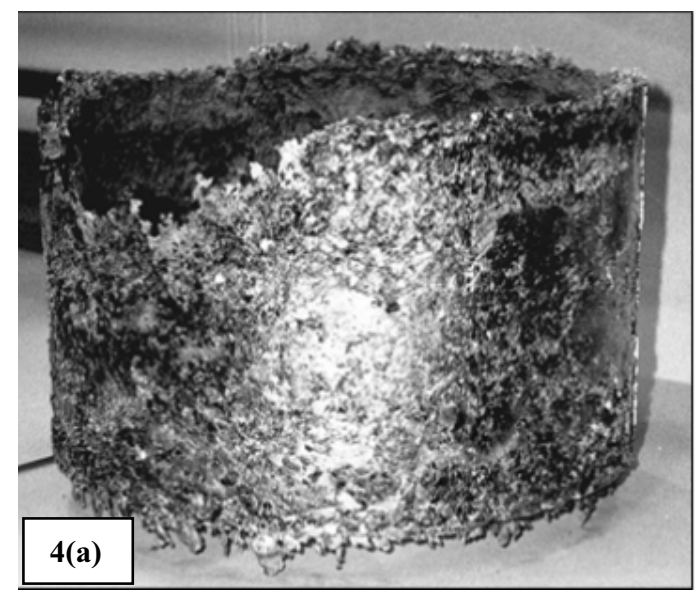

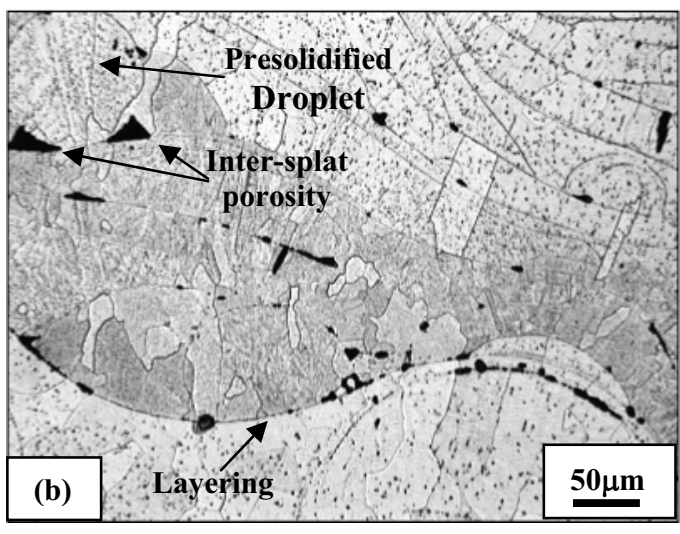

Fig. 4. (a) Photograph of ring produced under cold deposition conditions, and (b) typical corresponding layered microstructure.

Fig. 5(a) is a secondary electron micrograph of a region taken from the preform produced under cold conditions showing the particle linear arrangements and inter-splat/droplet boundaries. EDX analysis of the interlayer boundary particles showed an average $\mathrm{Nb}$ concentration of $\sim 6 \mathrm{wt} \%$, suggesting the presence of grain boundary $\delta$ phase [5]. However some of the particles showed much higher $\mathrm{Nb}$ concentrations, such as the particle concentrations shown in Fig. 5(b), and in this case, these particles were ascribed to $\mathrm{Nb}$ and $\mathrm{Ti}$ rich carbides. It is postulated that carbides and $\delta$ precipitates decorate interlayer boundaries under these deposition conditions.
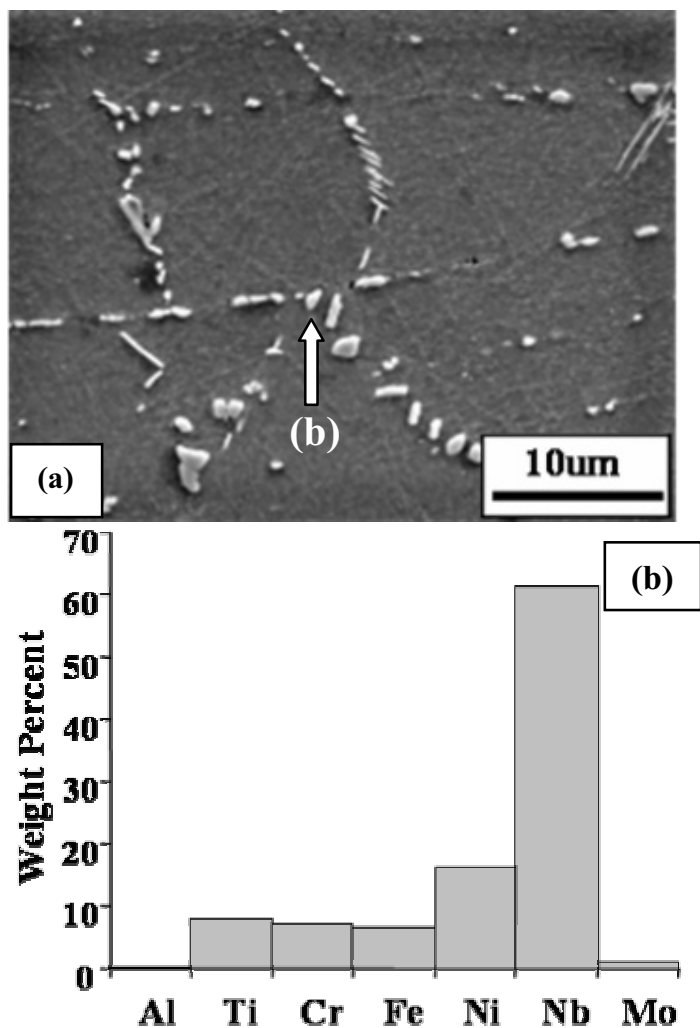

Fig. 5. (a) Secondary electron image of layering observed in ring manufactured under cold deposition conditions, and (b) composition of a typical interlayer particle shown in (a). 
$\underline{\text { Intermediate Deposition Conditions }}$

Fig. 6(a) shows a preform manufactured under intermediate deposition conditions with excellent shape and a satisfactory surface finish for further processing. The corresponding microstructure shown in Fig. 6(b) consisted of equiaxed grains, fine-scale $\delta$ phase and $\mathrm{Nb}$ and $\mathrm{Ti}$ rich primary carbides, and significantly less or no layering. Porosity fractions were reduced to $\sim 1.0$ area $\%$ and the typical pore size was $<10 \mu \mathrm{m}$. Pores occurred primarily in the inter-splat regions at the outer surface of the preform with occasional shrinkage pores at grain boundaries.

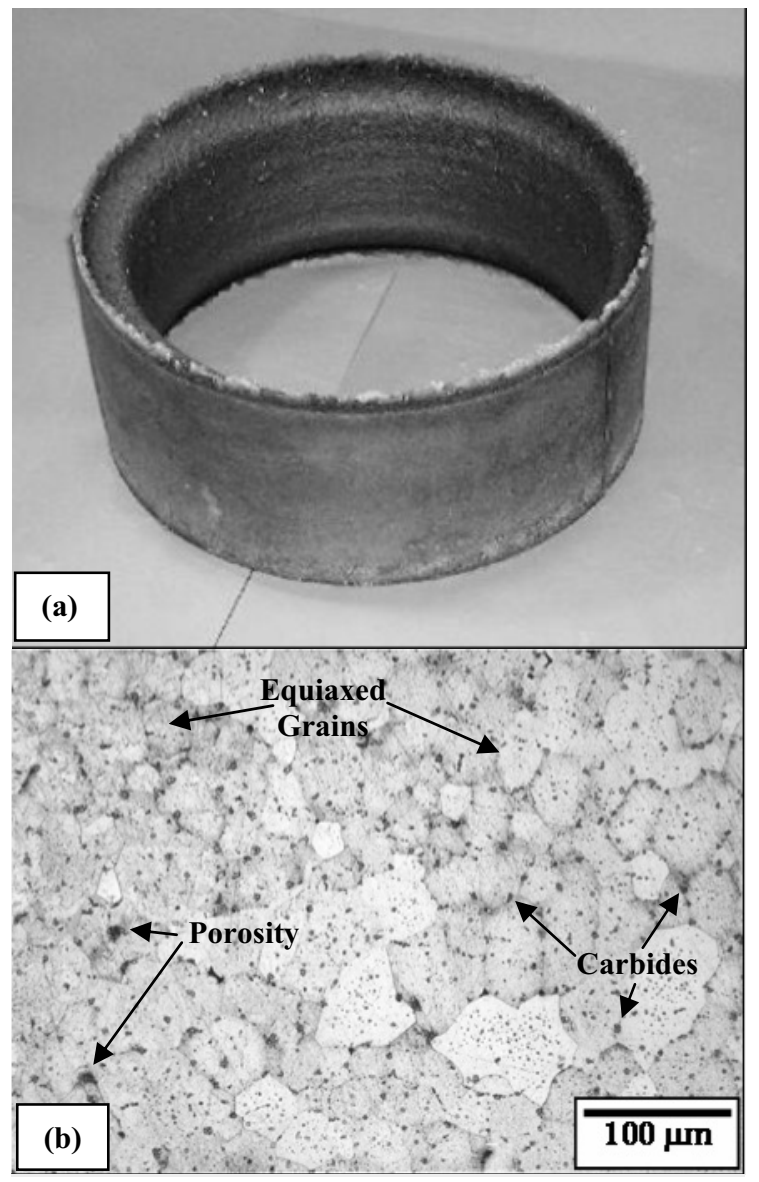

Fig. 6. (a) Photograph of a ring produced under intermediate deposition conditions, and (b) a typical corresponding microstructure.

\section{$\underline{\text { Microstructure of CSD IN718 After Heat Treatment }}$}

As-sprayed material from the IN718 preform produced under intermediate conditions was solution treated and double aged according to the conditions described in the experimental section. Heat treatment had no significant effect on the average grain size which remained at $\sim 36 \mu \mathrm{m}$, although there was a slight reduction in the amount of intragranular $\delta$ phase. However, coarse acicular precipitates were now evident at grain boundaries and triple points, as shown in Fig. 7(a). To identify these phases, EDX microanalysis was carried out on the grain boundary cluster shown in Fig. 7(b). Fig. 7(c) shows elemental concentrations at the positions specified in (b), indicating that $\mathrm{Nb}$ concentrations reached $\sim 19 \mathrm{wt} \%$ with an average of $8 \mathrm{wt} \%$. This supports the identification of $\delta$ phase that had coarsened during heat treatment in the presence of microsegregated $\mathrm{Nb}$ at the grain boundaries. The highest $\mathrm{Nb}$ concentration was accompanied by an increase in $\mathrm{Ti}$ and a decrease in $\mathrm{Cr}$ and $\mathrm{Fe}$ concentrations, suggesting a $\mathrm{Nb}$ and $\mathrm{Ti}$ rich primary carbide situated between the acicular $\delta$ precipitates.
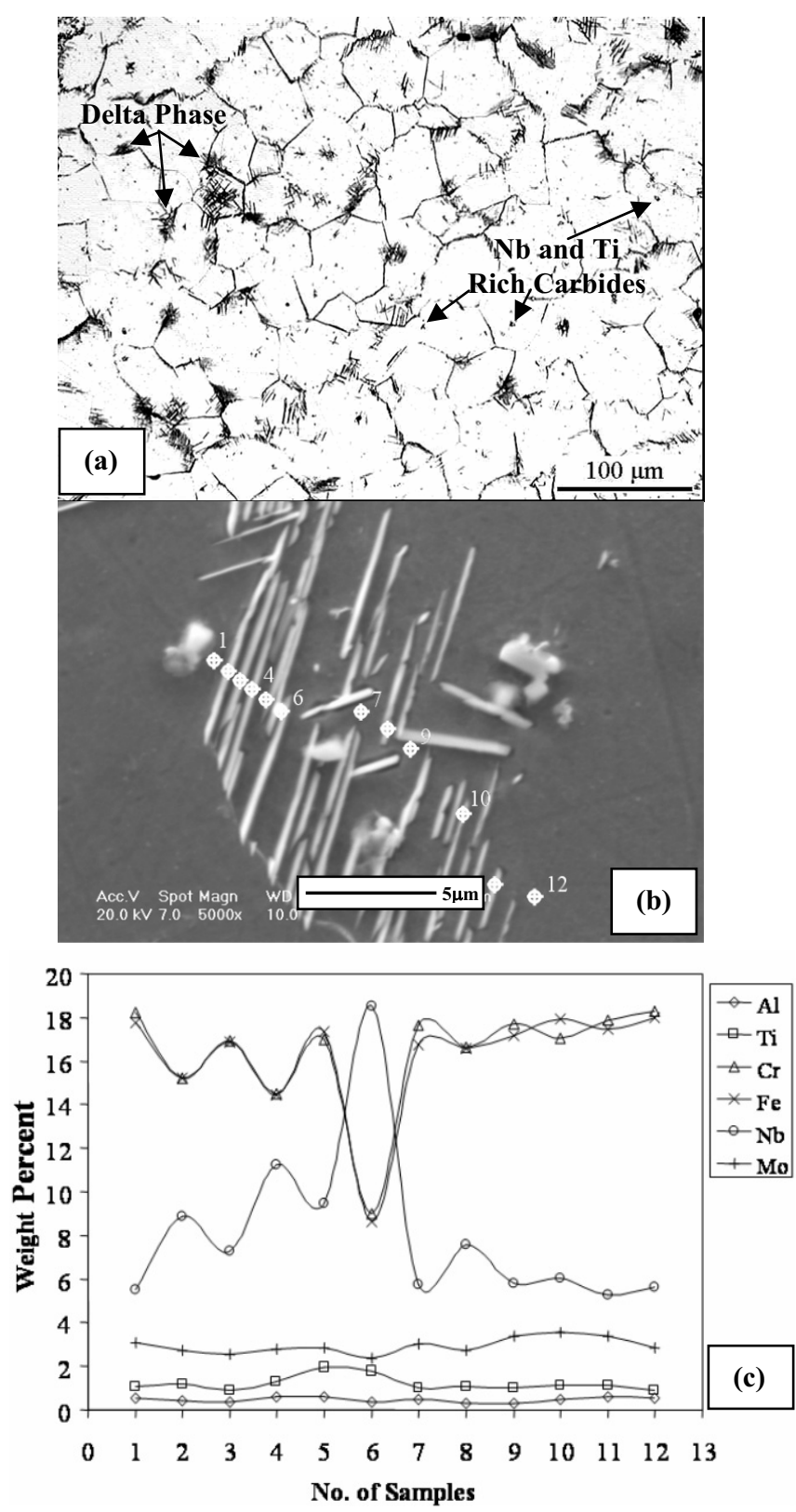

Fig. 7. (a) The microstructure of standard heat treated CSD IN718, (b) secondary electron image of coarse acicular precipitates at the grain boundary of heat treated CSD IN718, and (c) elemental concentrations at the locations shown in (b).

The presence of coarse acicular $\delta$ phase prompted the investigation of a higher temperature solution treatment to dissolve $\delta$ precipitates and to promote $\mathrm{Nb}$ homogenisation. Using 
an initial solution treatment of $1040^{\circ} \mathrm{C}$ for $1 \mathrm{hr}$ followed by the standard heat treatment gave little grain growth and the resulting microstructure shown in Figs. 8(a) and 8(b) comprised fine-scale carbides, carbonitrides, and equiaxed grains. Delta phase was again evident as globular precipitates distributed along grain boundaries.

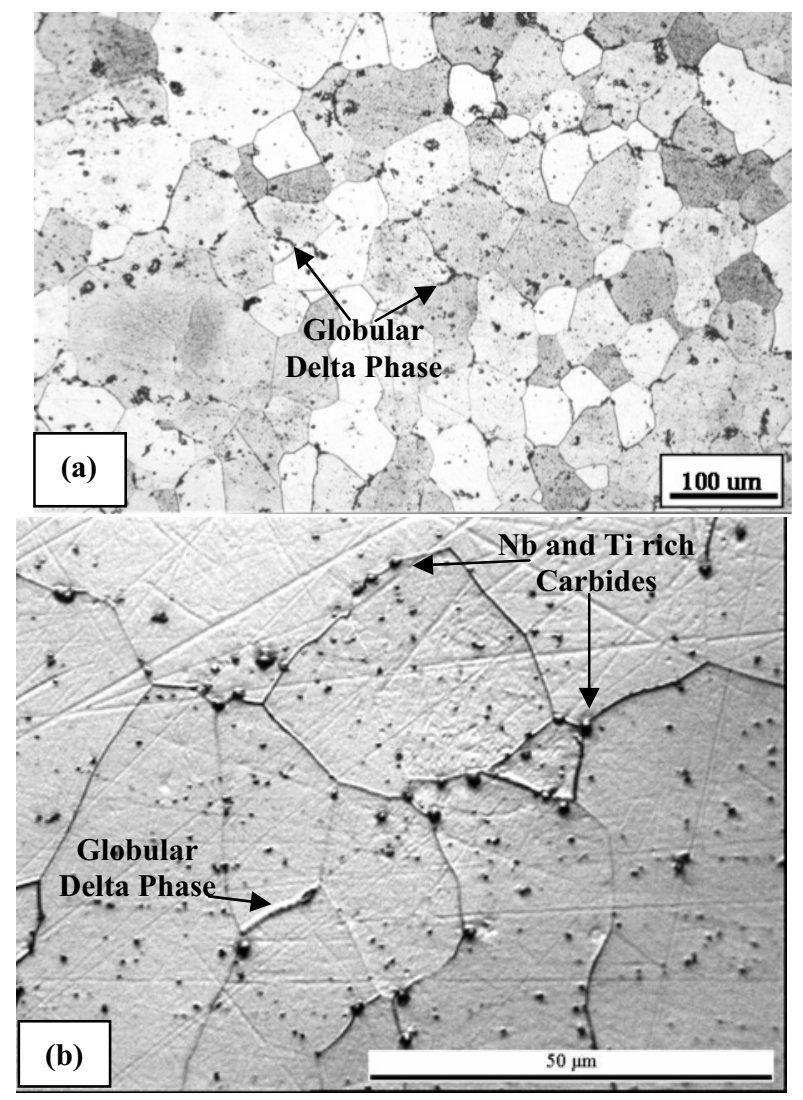

Fig. 8. (a) Microstructure of CSD IN718 after solution treatment at $1040^{\circ} \mathrm{C}$ then standard heat treatment, and (b) the same microstructure at higher magnification.

\section{Numerical Modelling of Heat Flow}

In order to optimise deposition conditions and to understand microstructural development a numerical model was developed to investigate the heat flow in the mild steel substrate and developing Ni preform during CSD. The model considered different heat transfer mechanisms such as convection and radiation operating at the interfaces and boundaries of the forming ring and the substrate. On the outside surfaces of the ring and the substrate, heat was removed by radiation and convection. An interfacial heat transfer coefficient governed the rate of heat exchange between the ring and the substrate. Within the ring, a heat source was required to account for the release of the latent heat of solidification. The general heat conduction equation is:

$$
\rho c \frac{\partial T}{\partial t}=\nabla \cdot(k \nabla T)+\dot{Q},
$$

where $T$ is temperature, $t$ is time, $\rho$ is density, $c$ is specific heat, $k$ is thermal conductivity, and $\dot{Q}$ is heat generation rate per unit volume. To account for the release of latent heat during solidification, an apparent heat capacity method was used in which $\dot{Q}$ was related to the solidified fraction $f_{s}$ as follows:

$$
\dot{Q}=\rho L \frac{\partial f_{s}}{\partial t}=\rho L \frac{\partial f_{s}}{\partial T} \frac{\partial T}{\partial t},
$$

where $L$ is the latent heat. By the apparent heat capacity method:

$$
c^{\prime}=c-L \frac{\partial f_{s}}{\partial T},
$$

hence

$$
\rho c^{\prime} \frac{\partial T}{\partial t}=\nabla \cdot(k \nabla T)
$$

An explicit finite difference (FD) method was used to solve the heat conduction equation and 2D cylindrical axi-symmetry was assumed.

Fig. 9 shows measured ring and substrate temperatures obtained by embedded thermocouples for typical CSD conditions, together with modelled thermal histories from the corresponding locations in the computational FD grid. For this set of boundary conditions, modelled temperatures were in good agreement with the experiment. However, more experimental data are required in order to define boundary conditions rigorously for all CSD conditions.

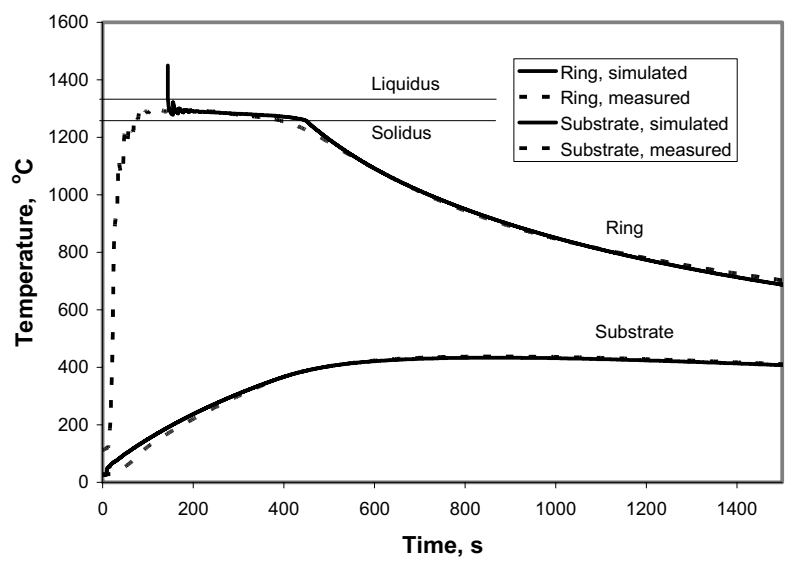

Fig. 9. Comparison of modelled temperatures with experimental results, for both the ring and the substrate.

Fig. 9 also shows that superheated liquid droplets were cooled very quickly into the mushy zone between the alloy solidus and liquidus temperatures. Under other CSD conditions the superheated droplets may be cooled to complete solidification. As expected, local cooling rates were slower in the mushy zone than below the solidus because of the release of the latent heat. Complete solidification took $450 \mathrm{~s}$ and the total deposition time was $345 \mathrm{~s}$. Once fully solidified, the temperature gradients within the preform gradually reduced. The mild steel substrate temperature gradually increased from room temperature throughout deposition up to $\sim 430^{\circ} \mathrm{C}$ after $800 \mathrm{~s}$ as heat was transferred from the spray formed preform to the steel substrate.

Figs. 10(a)-(c) show contour maps of the time taken for complete solidification for the three experimental CSD conditions described earlier broadly as hot, cold and intermediate respectively. Solidification times were $<78 \mathrm{~s}$ irrespective of position under the cold conditions in (b). Whereas under hot conditions in (a), there was still significant liquid fraction in the preform at the end of 
deposition after $345 \mathrm{~s}$, as confirmed by Fig. 11 that shows the solid fraction distribution at the instant that deposition ended. Under these conditions, the preform is expected to have insufficient integral strength and slumping will occur, as shown experimentally in Fig. 2(a). For intermediate conditions in (c), a liquid fraction persists beyond the end of deposition, but in this case a sufficient thickness of high solid fraction material had developed at the ring surfaces to inhibit slumping while ensuring there was some re-melting between successive layers of deposited material associated with passes of the spray.
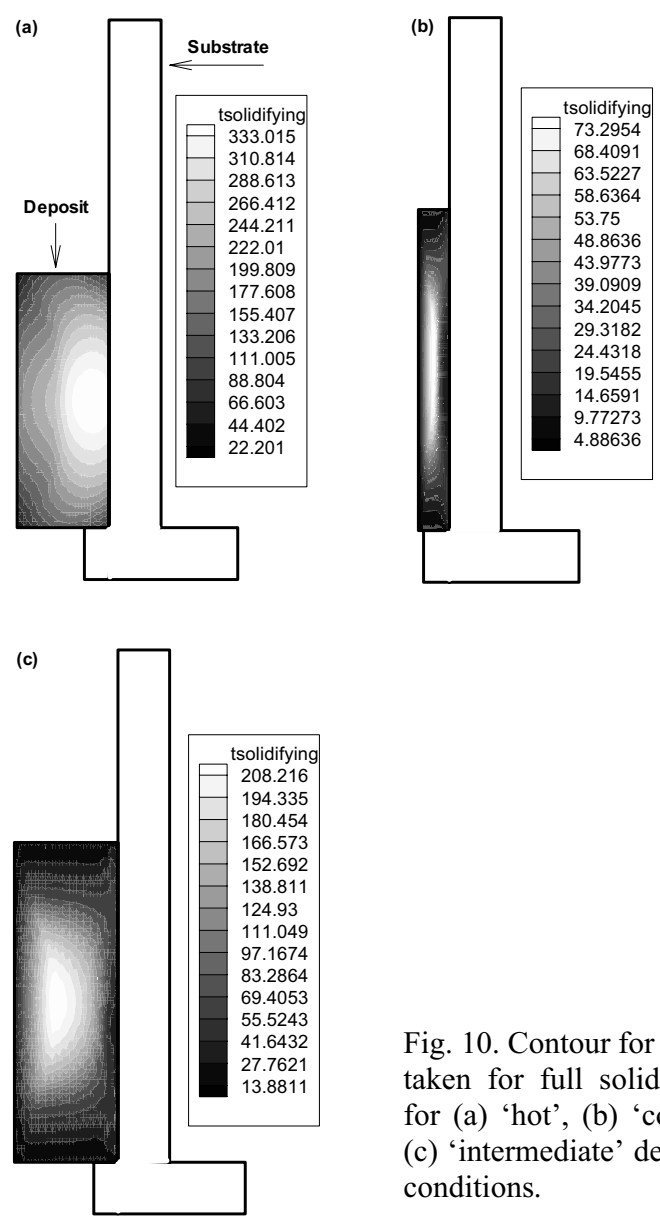

Fig. 10. Contour for the time taken for full solidification for (a) 'hot', (b) 'cold' and (c) 'intermediate' deposition conditions.

Fig. 11. Contour of solid fraction in the 'hot' deposited ring at the end of the deposition.
The re-melting behaviour is described in more detail in Fig. 12 that shows the thermal history of three points in three spray formed IN718 preforms as a function of the layer thickness deposited in each pass, namely $0.4,0.8$ or $1.6 \mathrm{~mm}$. The modelled thermal histories showed repeated heating/cooling or even repeated melting/solidifying cycles immediately after deposition because of the continuing intermittent heat input from subsequent deposition passes. The first post-deposition pass of the spray had the most significant re-heating/re-melting effect. If just deposited layers were not re-heated to a temperature within the mushy zone by the first post-deposition pass, the bonding or microstructural fusion between layers is expected to be weak and a layered or unfused structure will form [7], as shown experimentally in Fig. 4(b). For a deposited layer thickness of $0.4 \mathrm{~mm}$, Fig. 12 shows that the previously deposited layers were reheated to above the solidus for only a very short time, and the microstructural evidence in Fig. 4(b) suggested there was insufficient fusing of the microstructure across inter-layer boundaries. As the layer thickness (mass flow rate) increased to 0.8 and $1.6 \mathrm{~mm} /$ pass, local solidification time increased and the persistence of liquid ensured good inter-layer melting and microstructural homogenisation, as shown in Fig. 6(b).

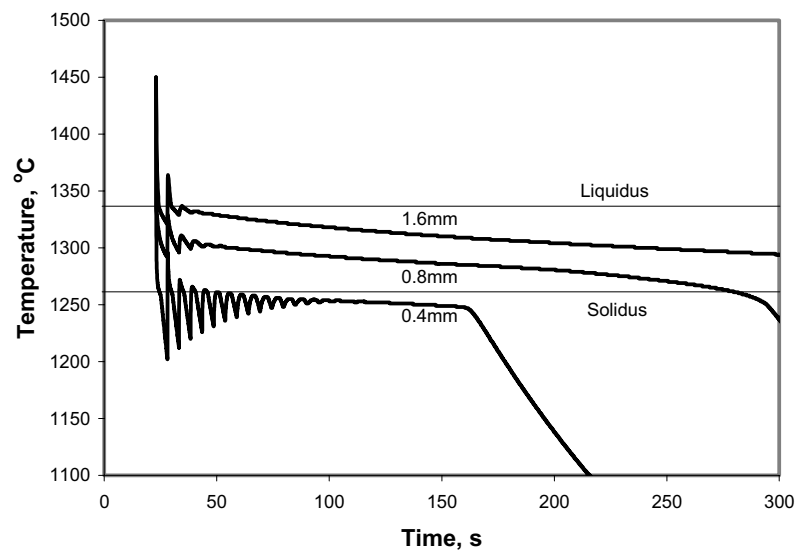

Fig. 12. IN718 thermal histories as a function of thickness per pass showing repeated heating/cooling behaviour.

\section{Discussion}

Experimental and numerical investigations confirm that changes in the mass flux of spray droplets arriving at the deposit surface, coupled with changes to the reciprocation pattern of the substrate, can have profound effects on the deposit quality and microstructural features. The mass flux conditions at deposition are in turn dependent on the mass flow rate at the periphery of the rotating cup and the size and heat content of the droplets created at this point by atomisation. A relatively high mass flow rate and slow reciprocation speed creates a thick, semi-solid layer on the surface, which produces a hot slumped deposit, whereas a comparatively low mass flow rate and faster reciprocation speed causes only partial inter-layer mixing of the deposited droplets, leading to a cold deposit microstructure. A low mass flow rate coupled with an appropriate reciprocation speed and axial displacement provides more fitting, intermediate deposition conditions. By inference, there is an optimum balance between local heat extraction and local heat input rates, as controlled by the local deposition conditions, and which can provide a near optimum microstructure. The concept of a global heat rate balance 
is also valuable in understanding thermal history and microstructural effects. For example, Fig. 13 shows two solid fraction contour plots at the instant of the end of the deposition. In both, the overall mass flow rate, deposition time, and consequently total deposited mass are identical. However in the second case, the reciprocation velocity is half of the first case so that the layer thickness doubles, and the total number of layers is halved. Despite these differences, the solid fraction contours are similar since changes in reciprocation velocity only do not affect the global heat rate balance. However, as shown in Fig. 12, the number of re-melting cycles will be different in the two cases and consequently microstructural differences may still arise. Note the last deposition location is lower left corner for the first case, and the upper left corner for the second case producing the local low solid fraction.


Fig. 13. Comparison of solid fraction contour plots for two set of deposition parameters.

Variations in the preform microstructure are primarily due to differing extents of $\mathrm{Nb}$ segregation. At one extreme, Laves phase forms in the interdendritic regions of hot deposits because the solidifying $\gamma$ matrix rejects the $\mathrm{Nb}$, Ti and Mo. Consequently, primary dendrites are enriched in $\mathrm{Ni}, \mathrm{Cr}$, and $\mathrm{Fe}$. If microsegregation leads to $\mathrm{Nb}$ concentrations in excess of $10 \mathrm{wt} \%$, then Laves will form; at higher concentrations of $\mathrm{Nb}$ and $\mathrm{Ti}$, carbides and carbonitrides will also form where sufficient $\mathrm{C}$ is present [5]. Significantly reduced $\mathrm{Nb}$ segregation results were achieved in CSD preforms at the expense of an unfused or banded microstructure associated with poor inter-layering and re-melting, as previously reported in smaller preforms [8]. Layering is characterised by insufficient melting of $\gamma$-dendrites and $\mathrm{Nb}$ and $\mathrm{Ti}$ rich carbides and carbonitrides. During cooling of the preform, $\delta$ forms alongside the carbides and carbonitrides wherever levels of $\mathrm{Nb}$ are sufficiently high $(>6.0 \%)$. When conditions are intermediate, the in-coming droplets either arrive before the underlying layer has fully solidified or cause it to remelt due to the additional enthalpy they provide. Hence, the interlayer boundary is effectively removed as the liquid combines at the surface $[9,10]$. This leads to a uniform structure consisting of equiaxed grains and fine-scale carbides, carbonitrides, and $\delta$ precipitates.
To achieve the required microstructure and mechanical properties, heat treatments are required to control precipitation of the various phases in IN718. Precipitation of these phases is dependent on the distribution of $\mathrm{Nb}$, the heat treatment temperature and the time at temperature. Following a standard heat treatment, coarse acicular $\delta$ precipitates formed at grain boundaries and triple points in intermediate deposition conditions, because of $\mathrm{Nb}$ microsegregation. Using a solution treatment temperature increased to $1040^{\circ} \mathrm{C}$, the upper limit of the delta solvus, led to a $\mathrm{Nb}$ distribution sufficiently homogenised to prevent the formation of acicular $\delta$ precipitates. Subsequent solution treatment at $980^{\circ} \mathrm{C}$ followed by double ageing promoted the formation of globular $\delta$ which aids grain size control and improves mechanical properties [11].

\section{Conclusions}

- Ring-shaped IN718 preforms have been produced successfully using centrifugal spray deposition.

- The microstructure of IN718 preforms was controlled by the atomisation and deposition parameters, in particular the mass flow rate, and the rate and extent of substrate motion.

- A broad range of process conditions spanning typical hot, cold, and intermediate deposition conditions gave rise to a range of characteristic macrostructures and microstructures that under near optimum conditions led to a good surface finish, low porosity, reduced banding and inter-layer precipitates, and an equiaxed grain morphology.

- The as-sprayed microstructure benefited from a modification to standard heat treatment procedures, and produced a microstructure comparable to cast/wrought equivalents.

\section{Acknowledgements}

The authors would like to thank the UK Engineering and Physical Sciences Research Council and DSTL for financial support; Doncasters plc, Siemens, Bodycote Ltd, and Qinetiq for technical support; and Mr. M P Glynn for technical expertise.

\section{References}

[1] A.R.E. Singer and S.E. Kisakurek, "Centrifugal Spray Deposition of Aluminium Strip", Metals Technology, (1976), 565570.

[2] A.L. Dowson, M.H. Jacobs, M.D. Barratt, "Centrifugal Spray Deposition - An Alternative Route for the Manufacture of Ring Components", Proceedings of the International Conference on Powder Metallurgy and Particulate Materials, New Orleans, USA, (2001).

[3] D. Whyman, J.M. Young, M.H. Jacobs, "Processing and Evaluation of Waspaloy Ring Preforms Prepared by Centrifugal Spray Deposition", Proceedings of Fourth Intl. Conference on Powder Metallurgy in Aerospace, Anaheim, CA, USA, (1995), 147-154.

[4] M.H. Jacobs and J.M. Young, "Primary Plasma Melting of Aerospace Alloys and Their Secondary Processing by Spray Forming and Gas Atomisation", AVS Intl. Symposium on Liquid Metals Processing \& Casting, Sante Fe, New Mexico, USA, (1994). 
[5] J.F. Radavich, "The Physical Metallurgy of Cast and Wrought Alloy 718”, Superalloy 718 - Metallurgy and Applications, TMS, (1989).

[6] J.J. Schirra, R.H. Caless, R.W. Hatala, "The Effect of Laves Phase on the Mechanical properties of Wrought and Cast + HIP Inconel 718", Superalloys 718, 625, and Various Derivatives, Loria, E.A, editor. TMS (1991), 375-388.

[7] K.H. Baik, P.S. Grant, B. Cantor, "The equiaxed-banded microstructural transition during low pressure plasma spraying", Acta Materialia, 52 (2004), 199-208.

[8] M.K. Hedges, A.P. Newbery, P.S. Grant, "Characterisation of electric arc spray formed Ni superalloy IN718", Materials Science and Engineering A, A326 (2002), 79-91.

[9] B. Cantor, K.H. Baik, P.S. Grant, "Development of Microstructure in Spray Formed Alloys", Progress in Materials Science, 42 (1997), 373-392.

[10] R.M. Ward, M.D. Barratt, M.H. Jacobs, A.L. Dowson, “A Simple Transient Numerical Model for Heat Transfer and Shape Evolution During the Production of Rings by Centrifugal Spray Deposition", Proceedings of the International Symposium on Liquid Metal Processing and Casting, Nancy, France, ASM international, (2003), 223-239.

[11] M.D. Barratt, A.L. Dowson, M.H. Jacobs, "The Microstructure and Properties of IN718 Rings Produced by Centrifugal Spray Deposition”, Proceedings of the Fifth International Conference on Spray Forming, Bremen, Germany, (2003), Part 5, 57-68. 